\title{
LAS ESTRATEGIAS DE INDONESIA FRENTE AL ASCENSO DE CHINA
}

Ezequiel Ramoneda*

Instituto de Relaciones Internacionales -

Universidad Nacional de La Plata

\er131079@gmail.com
Recibido: 24 noviembre de 2019

Aceptado: 3 de marzo de 2020

DOI: $10.46553 /$ colec.31.1.2020.p69-88

Resumen: El presente trabajo tiene por objetivo analizar la respuesta de la República de Indonesia durante el gobierno de Joko Widodo frente al desafío del ascenso regional de la República Popular China. El análisis propuesto se realizará considerando el ámbito marítimo entendiendo que es en éste donde se despliega más distintivamente la respuesta indonesia. Ahora bien, este ámbito marítimo no será enmarcado dentro de la concepción regional del Asia-Pacifico, sino dentro de la concepción regional del Indo-Pacifico, cambio que también responde a la reconfiguración del contexto geopolítico y geoeconómico como consecuencia del ascenso regional de China. La hipótesis asumida es que la estrategia de Indonesia no pretende competir o confrontar sino más bien adecuar y complementar la proyección hegemónica de China en la región.

Palabras claves: Indonesia; Pivote Marítimo Global; Indo-Pacífico; ASEAN; China

Abstract: The aim of this paper is to analyze the response of the Republic of Indonesia during the government of Joko Widodo in the face of the challenge of the regional rise of the People's Republic of China. The proposed analysis will be carried out considering the

\footnotetext{
* El autor es Licenciado en Estudios Orientales (USAL). Maestrando en Relaciones Internacionales (UNLP). Coordinador del Centro de Estudios del Sudeste Asiático, y Secretario del Departamento de Asia y el Pacífico (IRI-UNLP). Miembro del Grupo de Estudios sobre Asia y América Latina (IEALC-UBA). Secretario de la Sección Argentina de la Asociación Latinoamericana de Estudios de Asia y África. Participante del Grupo de Trabajo del Sudeste Asiático del Comité de Asuntos Asiáticos (CARI).
} 
maritime area, understanding that this is where the Indonesian response is most distinctively appreciated. However, this maritime area will not be framed within the regional concept of Asia-Pacific, but within the regional conception of the Indo-Pacific, a change that also responds to the reconfiguration of the geopolitical and geoeconomic context as consequence also of the regional rise of China. The hypothesis assumed is that Indonesia's strategy is not intended to compete or confront but rather to adapt and complement China's hegemonic projection in the region.

Keywords: Indonesia; Global Maritime Fulcrum; Indo-Pacific; ASEAN; China

\section{Introducción}

Las relaciones entre Indonesia y China han experimentado un gran desarrollo desde el gobierno del presidente Susilo Bambang Yudhoyono, en el cual se firmaron la Asociación Estratégica en abril de 2005, luego elevada a Asociación Estratégica Comprehensiva en noviembre de 2013. Actualmente, el gobierno del presidente Joko Widodo pretende hacer de Indonesia una potencia marítima a partir de su visión del Pivote Marítimo Global, lo que no sólo implica un cambio de paradigma de la política nacional del país, tradicionalmente de base territorial, a una más equilibrada, resaltando la base marítima, lo que se refleja en la orientación de la política exterior del país, sino también que coincide con el ascenso regional de China durante el gobierno del presidente Xi Jinping, entendido como el proceso más importante de reconfiguración del contexto geopolítico y geoeconómico regional del Asia-Pacifico.

El presente trabajo tiene por objetivo analizar la respuesta de la República de Indonesia durante el gobierno de Joko Widodo frente al desafío del ascenso regional de la República Popular China. El análisis propuesto se realizará considerando el ámbito marítimo entendiendo que es en éste donde se apreciar más distintivamente la respuesta indonesia. Ahora bien, este ámbito marítimo no será enmarcado dentro de la concepción regional del Asia-Pacifico, predominante en los últimos treinta años, sino dentro de la concepción regional del Indo-Pacifico, de planteamiento 
reciente, cambio que también responde a la reconfiguración del contexto geopolítico y geoeconómico como consecuencia también del ascenso regional de China. Más allá de lo anterior, esta última concepción también es más adecuada debido a que el archipiélago indonesio se ubica geográficamente entre el océano Índico y el océano Pacífico. La hipótesis asumida es que la estrategia de Indonesia no pretende competir o confrontar sino más bien adecuar y complementar la proyección hegemónica de China en la región.

El trabajo estará compuesto de la siguiente manera. Luego de la introducción, se presentará el marco teórico a partir del cual se analizará la estrategia indonesia y se analizará la visión marítima enunciada por el presidente Widodo. Luego de lo cual se presentará cuatro secciones donde se tratará respectivamente aspectos específicos de esta visión, a saber, la identidad, la seguridad, la diplomacia y la economía marítima, en función al ascenso regional de China. Finalmente se plantearán las conclusiones.

\section{Marco Teórico de Poder Marítimo}

La estrategia marítima de Indonesia se analizará bajo el marco teórico de las teorías de poder marítimo. Entre los precursores, se encuentra la obra “The Influence of Sea Power upon History (1660-1783)" de 1890 de Alfred Thayer Mahan. Mahan planteo la noción de uso del mar, pero sin terminar de definirla en relación al poder marítimo. Para Mahan, el poder marítimo de un país se ve afectado por los siguientes factores: 1) su posición geográfica, 2) su conformación física, 3) la extensión territorial, 4) su población, 5) el carácter nacional, y 6) el carácter de su gobierno. (Mahan 1890) Enumera así factores que remiten a atributos materiales de poder de un país, basados en la geografía y demografía, entre otros, como también a atributos subjetivos como la consciencia de un pueblo con las cuestiones marítimas. Para Mahan, la estrategia Naval tiene por objetivo crear, sostener e incrementar, tanto en tiempos de paz como en tiempos de guerra, el poder naval de un país (Mahan 1890).

Más cercanos en el tiempo, se destacan, entre otros, los aportes de Ken Booth y John Richard Hill. Con respecto al primero, en su obra "Navies and Foreign Policy” de 1977 Booth desarrolló la noción de usos del Mar a partir 
de la cual se determinan las funciones de las armadas, las que pueden ser concebidas como una trinidad. La característica de esta trinidad está definida por los tres modos característicos de acción a partir de los cuales las armadas realizan sus propósitos, a saber, el militar, el diplomático, y el policiaco. Al respecto, resalta que el modo de acción militar, es decir la capacidad de una armada para amenazar o usar la fuerza, es el que da significado a los otros modos de acción (Booth 2014). Con respecto al último en su obra "Maritime Strategy for Medium Powers" de 1986 Hill afirmó que el poder marítimo es la capacidad de usar el mar, y que entre sus funciones está la de proteger los intereses económicos del país. Aunque sea en grado mínimo, la población de un país que posea litoral marítimo aprovechará los recursos marinos en su beneficio. Esto puede resultar beneficioso para un Estado en términos económicos, de ahí que deba proteger los recursos marítimos de la economía, y proyectar fuerza contra sus oponentes (cit. en Solís Oyarzún 1998; 2002). Tomados en conjunto ambos autores, a partir del fundamento del aspecto militar, se destaca la importancia del aspecto diplomático y el aspecto económico respectivamente del poder marítimo.

Finalmente, se encuentra la obra "Seapower: A Guide for the TwentyFirst Century" de 2007 de Geoffrey Till, que en cierta manera es una síntesis actual de las teorías de poder marítimo. Till entiende por poder marítimo a la capacidad de un estado de influenciar el comportamiento de otros estados o actores en o desde el mar. Este es el producto de una amalgama de elementos constituyentes interconectados, los que les facilita o les dificulta a los países ser fuertes en el mar. Estos abarcan desde la población, la sociedad y el gobierno, hasta la tecnología, la geografía y la economía (Till 2013).

En resumen, a los fines de este trabajo se adoptará esta última definición de poder marítimo en tanto capacidad de un estado de influenciar el comportamiento de otros estados en o desde el mar, entendiendo que hay una serie de factores interrelacionados que condicionan el mismo, como los recursos materiales, la tecnología, la geografía y la población, a la vez que las áreas involucradas son no sólo la defensa, sino también la diplomacia y la economía. A partir de esta definición se entiende que la visión marítima para su país del presidente indonesio Joko Widodo (octubre 2014 - octubre 2019) busca desarrollar el kekuatan maritim o poder marítimo de Indonesia, 
a partir de la cual pretende convertirlo en el Poros Maritim Dunia o Pivote Marítimo Global (PMG), siendo un país marítimo soberano, avanzado, independiente y fuerte. Más allá de las afirmaciones en materia de política exterior que realizó durante los debates de la elección presidencial de octubre de 2014 (Connelly 2014), el presidente Widodo anunció internacionalmente su visión marítima de Indonesia en la IX Cumbre del Asia Oriental de noviembre de 2014. Esta visión fue profundizada en febrero de 2017, cuando el presidente Widodo emitió el decreto presidencial $\mathrm{n}^{\mathrm{o}} 16$ sobre la Kebijakan Kelautan Indonesia o Política Marítima de Indonesia, a partir del cual se buscó coordinar y orientar de manera más comprehensiva los programas e iniciativas relacionados con el sector marítimo del estado indonesio. De esta manera, a los cinco pilares originales del PMG enunciados en 2014 se agregaron dos más en 2017, para componer los actuales siete pilares sobre los cuales se construye el poder marítimo de Indonesia. Estos son 1) revivir una cultura marítima, 2) mejorar el desarrollo de los recursos marítimos, 3) desarrollar la economía, facilitar el comercio y la infraestructura marítima, 4) desplegar una diplomacia marítima, 5) reforzar la defensa naval y la seguridad marítima, 6) reforzar la protección ambiental y la administración del espacio natural marítimo, y 7) establecer instituciones y mejorar la gobernanza marítima (Anwar 2018; Laksmana 2017; Santoso y Nafisah 2017; Scott 2019; Shekhar 2018).

Es a partir del desarrollo del poder marítimo de Indonesia por medio de la implementación de la visión marítima de PMG que hay entender la respuesta de Indonesia frente al desafío del ascenso de China.

\section{La Identidad Marítima de Indonesia y el ascenso de China}

Durante el gobierno del presidente Suharto, el ejército tuvo el dominio de la política del país, incluso de la política exterior, lo que condujo a que las prioridades del gobierno y la asignación de recursos financieros en materia de defensa se centraran en cuestiones terrestres. Por el contrario, el desarrollo de cuestiones marítimas, y de la armada, fueron relegados en importancia. Con el retorno de la democracia y la resignificación de las relaciones cívico-militares tras el fin del gobierno de Suharto a finales de la década de 1990, y el creciente impulso en desarrollo económico del país a 
principios del nuevo milenio, que permitió un incremento del presupuesto de defensa, se comenzó a prestar mayor atención a las cuestiones marítimas, y junto con estas al rol de la armada en materia de defensa. Una de las cuestiones a tratar fue la identidad marítima del país, uno de los elementos fundamentales del poder marítimo.

Durante el gobierno de Susilo Bambang Yudhoyono (octubre 2004 octubre 2014) estableció en 2007 el Consejo Marítimo de Indonesia, una entidad consultiva para asistir en la elaboración de una regulación integral para la administración y el desarrollo de las cuestiones marítimas del país. Para este momento había diversas leyes que asignaban prerrogativas y obligaciones particulares sobre cuestiones marítimas dispersas en diferentes ministerios y agencias gubernamentales, con poca o nula articulación entre estas. Recién en octubre de 2014, poco antes de dejar el cargo, el presidente Yudhoyono firmó la ley $\mathrm{n}^{\circ} 32$ sobre los Undang-Undang Tentang Kelautan o los Asuntos Oceánicos, con la cual se reunió bajo una misma regulación elementos dispersos relacionados con la administración y el desarrollo de las cuestiones marítimas del país. También elevó a categoría de Ministerio al Consejo de Asuntos Marítimos y Pesca (Elisabeth 2018). Además en enero de 2008 emitió el decreto presidencial no 7 sobre Tentang Kebijakan Umum Pertahanan Negara o las Nuevas Pautas Generales sobre la Política de Defensa del Estado Indonesio, marco a partir del cual se pretendió avanzar en la modernización de las Tentara Nasional Indonesia o las fuerzas militares nacionales, mejorando sus capacidades, para constituir en 2024 una Kekuatan Pokok Minimum o Fuerza Esencial Mínima (MEF). En el mismo se atendió no sólo a las Angkatan Darat o las fuerzas terrestres, es decir el ejército, sino también a las Angkatan Laut o las fuerzas marítimas, es decir la armada. (Anwar 2018, Laksmana y Supriyanto 2018) Como una última cuestión, vale mencionar que en 2011 lanzó el Masterplan Percepatan Perluasan Pembangunan Ekonomi Indonesia o el Plan para la Aceleración y la Expansión del Desarrollo Económico de Indonesia (MP3EI) para desarrollar la infraestructura del país, incluida la marítima.

Ahora bien, fue el presidente Widodo quien elevó las cuestiones marítimas, no sólo en materia de defensa, sino también diplomáticas y económicos, a prioridad nacional, lo que se refleja en el anunció de su visión marítima. (Anwar 2018) Al respecto, se puede plantear que hay un mantenimiento del proceso de modernización de las fuerzas militares a 
partir del MEF, aunque más bien optimizando su desarrollo (Iis y Adhi 2015). A su vez, también hay una cierta continuidad entre el MP3EI del presidente Yudhoyono y el PMG del presidente Widodo, sólo que este último puso el énfasis en el sector marítimo (Sriyanto 2018). Para implementar su visión marítima, el presidente Widodo estableció el Ministerio Coordinador de Asuntos Marítimos, mecanismo institucional para supervisar, coordinar y orientar de una manera más coherente las funciones en cuestiones marítimas del Ministerio de Asuntos Marítimos y Pesca, el Ministerio de Transporte, el Ministerio de Energía y Recursos Minerales, y el Ministerio de Turismo, además de agencias gubernamentales. Según Shekhar, la narrativa de poder marítimo combina los objetivos de ambas la declaración de Djuanda y la doctrina de Wawasan Nusantara (Shekhar 2018). La Declaración de Djuanda fue adoptada en diciembre de 1957, donde se enunció por primera vez el término de Wawasan Nusantara o el carácter archipelágico del estado indonesio. Esta fue reconocida internacionalmente luego en la Convención de las Naciones Unidas sobre el Derecho del Mar de diciembre de 1982. Indonesia es el más grande del mundo con más de diecisiete mil quinientas islas. Antes de esta declaración, el territorio del archipiélago indonesio se limitaba a las islas, mientras que las aguas entre estas se consideraban como aguas internacionales (Arif y Kurniawan 2017; Laksmana y Supriyanto 2018). Esta declaración estableció que las aguas entre las diversas islas ubicadas dentro de las líneas de base rectas que conectan los puntos extremos de las islas más externas que componen un archipiélago constituyen jurídicamente aguas interiores del territorio indonesio y las líneas de base son los bordes marítimos internacionales de una entidad unificada de espacios marítimos y terrestres sujeta a la exclusiva soberanía del estado (Anwar 2018; Sebastián, Supriyanto y Arsana 2015; Shekhar 2018). De esta manera se consolido jurídicamente la integridad territorial del estado indonesio.

Además de lo anterior, el presidente Widodo buscó no sólo consolidar el desarrollo de los atributos de poder marítimos actuales del estado indonesio, sino también reforzar la identidad nacional como un país marítimo del pueblo indonesio, arraigada en su historia, su cultura y sus cosmovisiones marítima. Los bordes marítimos internacionales del estado archipelágico indonesio sirven para separar a la nación Indonesia de otras naciones circundantes. Todavía más, remite a la relación entre tanah o tierra y air o 
agua para el pueblo indonesio. El mar no separa, sino que une a las islas del archipiélago, superando las distancias geográficas, $\mathrm{y}$, por lo tanto, acerca a los pueblos que las habitan, superando las diferencias culturales que los distinguen. Tanah-air o tierra-agua es sinónimo del término patria (Ford y Lyons 2009).

Al respecto de esto, recuperó la historia de reinos marítimos del archipiélago indonesio. El presidente Widodo hizo referencias en distintas ocasiones a los grandes reinos de Sri Vijaya (siglo VII - siglo XIV), surgido en el centro de la isla de Sumatra, y de Mahapahit (siglo XIII-siglo XVI), surgido al este de la isla de Java, los cuales dominaron y/o influenciaron sobre grandes extensiones del espacio marítimo de la región insular del Sudeste Asiático, controlando las rutas comerciales que cruzaban los mares de la región (Santoso y Nafisah 2017). Fue con la llegada de los reinos europeos en el siglo XVI, que la influencia de los reinos marítimos indonesios empezó a verse obstruida, y con la ocupación colonial del archipiélago por el imperio holandés, ésta quedo prácticamente anulada. El dominio colonial holandés se orientó al control territorial, orientación que impuso la preeminencia de las cuestiones terrestres por sobre los marítimas. Esta orientación se mantuvo durante los gobiernos independientes de Sukarno y, especialmente, de Suharto, abarcando así prácticamente todo el siglo XX. Al rememorar la importancia regional de los grandes reinos marítimos de Sri Vijaya y Mahapahit, más allá de las diferencias que estos pudiesen tener con la actual Indonesia (entre las que cabe destacar la no correspondencia territorial exacta entre los primeros y la última, ya que los primeros extendieron su control sobre secciones de territorios que hoy corresponde a otros países del Sudeste Asiático, como Malasia y Filipinas, o los primeros tuvieron otras formas de gobierno, al ser monarquías, mientras la última es una república, o profesaban religiones diferentes, al adscribir los primeros a creencias hindú-budistas, mientras que actualmente la gran mayoría de la población indonesia profesa el Islam), el presidente Widodo plantea de manera sugerente esa vinculación de su visión marítima del PMG con la historia de esos grandes reinos marítimos del archipiélago indonesio, alentando a los indonesios a imaginarse a sí mismos como una potencia marítima. Historia que viene a contrastar la versión china, la cual plantea que las aguas de lo que actualmente se conoce como los Mares del Sur de China son aguas sobre las cuales China argumenta tener derechos 
históricos y sobre lo cual fundamenta la línea de nueve guiones, desconociendo la historia marítima de los otros países de la región.

\section{El aspecto militar del Pivoté Marítimo Global y el ascenso de China}

Planteado la cuestión identitaria de la visión marítima del PMG, se pasará a continuación a tratar sus aspectos militar, diplomático y económico respectivamente.

En lo que concierne a la cuestión de la keamanan maritim o seguridad marítima, la visión marítima del PMG se articula con la doctrina vigente de la armada indonesia, Eka Sasana Jaya u "orientación unificada para la victoria en el mar", la cual resalta a la defensa naval, la seguridad marítima y la diplomacia marítima como las principales tareas de la armada (Arif y Kurniawan 2017; Laksmana 2014). Al respecto, las autoridades indonesias buscan atender no tanto los desafíos de seguridad externos y amenazas tradicionales, sino más bien a los desafíos de seguridad internos, como conflictos potenciales en las islas más externas del archipiélago para asegurar la unidad nacional y la integridad territorial, y las amenazas no tradicionales, principalmente las actividades ilegales dentro de las aguas interiores del archipiélago, como la pesca ilegal y la piratería, además del terrorismo, para proteger los recursos económicos marítimos y garantizar la seguridad de la navegación. Es decir, el eje está en la seguridad marítima, sin una clara pretensión de proyección naval regional. Ahora bien, esto no implica que la armada no pueda responder de manera disuasiva a agresiones exteriores o violaciones a la Zona Económica Exclusiva de Indonesia (Shekhar 2018).

El gobierno indonesio está llevando adelante una modernización de la armada, buscando convertirla en una armada de aguas verdes, para proteger las aguas interiores y los bordes marítimos del archipiélago. A la fecha, entre los aspectos más destacados se encuentra el reacondicionamiento de seis fragatas clase Van Speikk-class fabricadas a mediados la década de 1960 en Holanda y compradas en el transcurso de la década de 1980, y la adquisición de cuatro corbetas SIGMA versión 9113 comprada al mismo país europeo. También el reacondicionamiento de los dos submarinos de la clase Cakra de tipo 209 por Corea del Sur, y la adquisición de tres submarinos diesel- 
eléctricos clase Chang Bogo de tipo 209 comprados al mismo país asiático; el tercero de estos a ser construido en territorio indonesio. Ahora bien, estas nuevas adquisiciones vienen a reemplazar más que a fortalecer la flota, ya que gran parte de sus componentes fueron comisionados durante la Guerra Fría, estando próxima a cumplir su vida útil (Schreer 2013; Schreer 2015; Scott 2019). Además, el gobierno indonesio dispuso pasar de dos a tres comandos de las flotas navales, distribuidos en Tanjung Pinang en las Islas Riau, Makassar en Sulawesi, y Sorong en Papua Occidental, dispuestos en función de los tres rutas marítimas que cruzan el archipiélago de norte a sur respectivamente a través de los estrechos de Malaca, Sunda y Lombok. (Laksmana 2014; Sebastián, Supriyanto y Arsana 2015)

Con respecto a China, el gobierno indonesio percibe con seriedad las crecientes tensiones marítimas por reclamos de soberanía que mantiene con otros países de la región del Sudeste Asiático, en lo que se conoce como los Mares del Sur de China (o los Mares del Este de Vietnam, o los Mares del Oeste de Filipinas), las cuales generan un ambiente hostil y pueden potencialmente desembocar en el estallido de un conflicto armado entre los países involucrados, lo que afectaría la estabilidad de los mares de la región. Al respecto, Indonesia no mantiene reclamos de soberanía con China, pero si han surgido tensiones recientes entre ambos países por incidentes en la Zona Económica Exclusiva de Indonesia alrededor de las aguas de las Islas Natuna de la provincia de las Islas Riau, conocidas desde julio de 2017 como el Mar del Norte de Natuna. La línea de nueve guiones demarcada por China, basada en supuestos reclamos históricos, pasa por las aguas de las Islas Natuna, yuxtaponiéndose sobre la Zona Económica Exclusiva de Indonesia. La zona de las islas Natuna es importante no sólo por las rutas comerciales marítimas que la cruzan, sino también por los ricos recursos piscícolas y los yacimientos de hidrocarburos, principalmente de gas natural, que se encuentran en el lecho marítimo. Ha habido enfrentamiento entre guardacostas indonesios y embarcaciones pesqueras chinas, que estaban pescando ilegalmente en la zona, protegidas por patrulleros chinos. Esto explica la decisión del gobierno indonesio de mejorar las instalaciones militares en las islas Natuna (Iis 2015; Iis y Adhi 2015; Laksmana y Supriyanto 2018; Scott 2019; Sebastián, Supriyanto y Arsana 2015; Shekhar 2018). 
A partir de lo planteado, desde un punto de vista militar, se puede afirmar que Indonesia no compite o confronta directamente frente a China en su proyecto hegemónico, por no tener pretensión de proyección naval regional. Más bien, atendiendo a la seguridad marítima de las aguas interiores del archipiélago y la protección de sus recursos en la Zona Económica Exclusiva, busca disuadir la presencia ilegal de embarcaciones civiles y militares chinas, lo que explica los incidentes surgidos, delimitados especialmente alrededor de las aguas de Islas Natuna.

\section{El aspecto diplomático del Pivoté Marítimo Global y el ascenso de China}

En lo que concierne a la cuestión de la diplomasi maritim o diplomacia marítima, el gobierno indonesio busca mediar en las disputas marítimas en los Mares del Sur de China entre diversos países de la región, reduciendo las tensiones y desactivando potenciales conflictos, para mantener la paz y la estabilidad. También pretende reafirmar la posición de Indonesia como eje en la región del Indo-Pasifik o el Indo-Pacífico.

Durante el gobierno de Yudhoyono, Indonesia impulso en 2010 la creación del Foro Marítimo de la ASEAN (AMF), y luego en 2011 el Foro Marítimo de la ASEAN plus (Elisabeth 2018). Fue durante su gobierno que se realizaron mediaciones entre los miembro de la ASEAN luego de que en julio de 2012 la organización no emitiera una declaración conjunta en su reunión de ministros de relaciones exteriores por primera vez en su historia, producto de las diferencias que tenían algunos miembros frente a los reclamos marítimos chinos en los Mares del Sur de China. (Camboya rechazo las presiones de Vietnam y Filipinas de incluir referencias más duras sobre los reclamos marítimos chinos). El Canciller indonesio, Marty Natalegawa viajó a Camboya, Vietnam y Filipinas, donde mantuvo reuniones con sus respectivos mandatarios, luego de lo cual se emitió un documento de seis principios. Tiempo después, en mayo de 2013 el Ministro Natalegawa propuso la iniciativa del "Pacifico Indo-Pacifico", basada en la firma de un Tratado de Amistad y Cooperación del Indo-pacifico. De cierta manera, busco mantener la centralidad de la ASEAN en la región del IndoPacífico. Fue presentada por el presidente Yudhoyono en la VIII Cumbre 
del Asia Oriental de noviembre de 2013, pero no logro prosperar (Roberts y Widyaningsih 2015; Scott 2019). No obstante, el gobierno de Widodo continuó en cierta medida con esta idea.

La Canciller indonesia, Retno Marsudi, comentó en enero de 2018 que el orden regional del Indo-Pacífico, además de ser libre y abierta, debe ser inclusiva, transparente, comprensiva, mutuamente beneficiosa, que asegure la paz y la estabilidad regional, basada en el derecho internacional, y centrada en la ASEAN (Shekhar 2018). Luego en la $32^{\circ}$ Cumbre de ASEAN de abril de 2018, el presidente Widodo propuso su estrategia de «Cooperación del Indo-Pacífico», la cual fue luego tratada en la reunión de ministros de relaciones exteriores de la organización en julio de ese año (Scott 2019; Shekhar 2018). Finamente en agosto de 2019 la ASEAN la adoptó en su Declaración sobre la Perspectiva de ASEAN sobre el IndoPacífico. Se trata de un nuevo intento de mantener la centralidad de la ASEAN en la región del Indo-Pacífico. De esto se desprenden dos cuestiones importantes. Primero, que, a diferencia de las visiones del IndoPacífico de Japón y los Estados Unidos, la visión del Indo-Pacifico de Indonesia/ASEAN incluye a China. Segundo, que Indonesia pretende ejercer un liderazgo regional y participar en las dinámicas del equilibrio de poder regional entre las grandes potencias, en consonancia con la posición internacional del país como una de las economías más importante del mundo, pero busca hacerlo no unilateralmente por fuera, sino participando dentro la arquitectura multilateral regional basada en la centralidad de ASEAN y los mecanismos de la ASEAN plus, incluida la Reunión de Ministro de Defensa de la ASEAN Plus (ADMM+), el Foro Regional de la ASEAN (ARF), y la ya mencionada Cumbre del Asia Oriental, entre otros. De esta manera busca hacer del Tratado de Amistad y Cooperación de la ASEAN y la participación en la Cumbre del Asia Oriental los aspectos fundamentales de un orden de facto del Indo-Pacífico, en donde participa China en tanto uno de los socios de diálogo de la ASEAN (Scott 2019). De ahí que Indonesia, más allá de sus capacidades reales, se apoya en la ASEAN para fortalecer su posición relativa frente a la de las grandes potencias, ampliando sus márgenes de maniobra internacionales o reduce normativamente la de las grandes potencias, contribuyendo positivamente a la aplicación de normas sobre cuestiones marítimas y mejorando la cooperación en defensa naval y seguridad marítima (Auliya y Sulaiman 
2019; Sriyanto 2018; Wright 2015). Así la ASEAN sirve de plataforma para que Indonesia realice su visión marítima de PMG en el Indo-Pacífico.

Ahora bien, la ASEAN es la principal organización, pero no la única, donde Indonesia busca avanzar sus intereses del Indo-Pacífico. Así, el gobierno indonesio ha buscado expandir su protagonismo diplomático en la Asociación de la Cuenca del Océano Indico (IORA). Se trata de un foro regional para impulsar la cooperación económica entre varios países litorales de la cuenca del océano Índico. Indonesia presidió este foro durante los años 2015-2017, coincidiendo este último año con el 20avo aniversario de la creación del mismo. Al respecto, en marzo de ese año organizó en Jakarta la primera Cumbre de la IORA para conmemorar este acontecimiento, y además propuso la firma de Carta de la IORA, también denominada la Carta de Jakarta, como contribución al foro (Aufiya 2017; Auliya y Sulaiman 2019; Scott 2019).

Desde un punto de vista diplomático, se puede afirmar que Indonesia no busca excluir, sino más bien incorporar a China dentro de la arquitectura multilateral regional del Indo-Pacífico basada en la centralidad de la ASEAN, para adecuar o restringir normativamente su comportamiento internacional, y posicionarse como mediador de las disputas marítimas en los Mares del Sur de China para mantener la paz y la estabilidad regional.

\section{El aspecto económico del Pivoté Marítimo Global y el ascenso de China}

Finalmente, en lo que concierne a la cuestión de la ekonomi maritim o economía marítima, el gobierno indonesio busca atraer inversiones extranjeras para desarrollar la infraestructura marítima del país para mejorar la conectividad inter-isleña, y acelerar a la vez distribuir mejor el desarrollo económico, recudiendo las brechas entre las islas del oeste y el este del archipiélago.

En este sentido, como se mencionó, el PMG de Widodo puede ser considerado como una reelaboración del MP3EI del Yudhoyono. Mientras que, en el marco del MP3EI, el proyecto más importante era Pendulum Nusantara o Péndulo Archipelágico, basado en el desarrollo del embarque de 24 puertos estratégicos del archipiélago, en el marco del PMG el proyecto 
más ambicioso es el Tol Laut o la Autopista Marítima, anunciado en noviembre de 2015, el cual incluye el desarrollo de las conexiones de 7 puertos estratégicos, a saber Kuala Tanjung y Belawan en Sumatra, Tanjung Priok y Tanjung Perak Java, Makassar y Bitung en Sulawesi, y Sorong en Papua, los cuales funcionarán como centro de carga internacionales, complementados con el desarrollo de las conexiones de decenas de puertos no comerciales para mejorar así el sistema de servicios logísticos del archipiélago (Negara y Das 2017; Sa'adah, Yakti y Susanto 2019; Shekhar 2018; Sriyanto 2018). También, el gobierno indonesio ha buscado complementar su desarrollo en infraestructura marítima con el Plan para la Conectividad de ASEAN (MPAC) de 2010, el cual se sustenta en parte en el Fondo de Inversión de ASEAN en conjunto con el Banco de Desarrollo Asiático. De 47 puertos considerados estratégicos de los miembros de la ASEAN, 14 de estos están ubicados en Indonesia, incluyendo los 7 de la Autopista Marítima (Damuri 2014; Sa'adah, Yakti y Susanto 2019).

Desde un punto de vista económico, se puede afirmar que Indonesia busca articular y complementar el desarrollo de la infraestructura de la visión marítima del PMG con las inversiones de la Iniciativa del Cinturón y la Ruta (ICR) de China, especialmente la Ruta Marítima de la Seda, anunciada durante la visita a Indonesia del presidente chino Xi Jinping en octubre de 2013, junto con el Fondo de Cooperación Marítima ChinaASEAN. Esto se basa en el concepto de gotong-royong o cooperación mutua (Anwar 2018; Negara y Das 2017; Scott 2019; Sriyanto 2018). Para Indonesia, China es una fuente de asistencia técnica y, por sobre todo, financiera para los programas de desarrollo de infraestructura. Para China, Indonesia es un socio estratégico de su iniciativa ya que las conexiones marítimas extra-regionales que pretende desarrollar pasan por jurisdicción indonesia. De esta manera la ICR puede ayudar a realizar la visión del PMG, mejorando las relaciones económicas entre ambos países. Entre los proyectos de inversiones de China en Indonesia en el marco de la ICR, destacan el puerto de Kuala Tanjung en Sumatra, el Aeropuerto Internacional de Lembeh en Sulawesi, y el tren de alta velocidad entre Jakarta y Bandung. Ahora bien, el gobierno indonesio no ha reducido a China su necesidad de inversiones, también aceptando inversiones de otros países, como Japón, y diversificando los proyectos en infraestructura 
otorgados. Desde esta manera, no cae en la dependencia económica, y la consecuente injerencia política, de un solo actor (Sriyanto 2018).

\section{Conclusión}

Al asumir la presidencia de Indonesia en 2014, el presidente Widodo anunció su visión marítima del PMG para desarrollar el poder marítimo del país. La misma puede ser considerada como una continuación a la vez que una reelaboración de iniciativas puestas en práctica por el antiguo presidente Yudhoyono. La principal diferencia está en que buscó articular las diversas cuestiones del sector marítimo de manera coherente, elevándolas a prioridad nacional. Ahora bien, hasta el momento se puede apreciar que la orientación de visión marítima del PMG es más bien de desarrollo interno, que proyección externa, teniendo un marcado perfil económico. El mar no solo conecta, sino que encierra riquezas. De esta manera el presidente Widodo busca impulsar el desarrollo económico del sector marítimo del país. Por lo tanto, no es la proyección de poder naval regional de Indonesia sino la defensa de sus intereses nacionales en función de su desarrollo económico lo que hay que tener presente en las estrategias llevadas adelante por Indonesia para responder al ascenso regional de China.

Se pueden identificar por lo menos cuatro estrategias para responder al ascenso regional de China. En principio, es importante destacar la recuperación que hace de la historia de reinos marítimos del archipiélago indonesio, que viene a contrastar con la versión china, en la que se fundamenta los supuestos derechos históricos de China en los Mares del Sur de China. Luego, desde la perspectiva militar, no existiendo reclamos de soberanía con China, la estrategia ha sido disuasiva, a partir del desarrollo de una armada de aguas verdes que permita proteger los recursos económicos en su Zona Económica Exclusiva, especialmente en el Mar del Norte de Natuna, frente al accionar ilegal de embarcaciones civiles y militares chinas en la misma. Desde la perspectiva diplomática, la estrategia ha sido fortalecer la arquitectura multilateral regional del Indo-Pacífico basada en la centralidad de la ASEAN para adecuar o restringir normativamente el comportamiento internacional, buscando reducir las tensiones que mantiene con otros países afectados en las disputas de los 
Mares del Sur de China para mantener la paz y la estabilidad regional, condiciones para su desarrollo económico. Y desde la perspectiva económica, mientras defiende sus recursos económicos en su Zona Económica Exclusiva, busca complementar el desarrollo de la infraestructura del PMG con las inversiones de la ICR, pero sin caer la dependencia económica al atraer también inversiones de otros países y diversificar proyectos.

Esto lleva a plantear que el gobierno de Joko Widodo ha buscado construir relaciones políticas más amistosas y económicas más profundas con la República Popular China, sin descuidar la defensa de los intereses nacionales del país, adecuando y complementando su visión marítima del PMG con la proyección hegemónica de China.

\section{Referencias}

Anwar, Dewi Fortuna. 2018. "The Emergence of Indonesia's Ocean Policy". PACNET 14: 1-2. Disponible en:

https://www.pacforum.org/sites/default/files/tmp/180222_PacNet_14.p df)

Arif, Muhamad y Yandry Kurniawan. 2017. "Strategic Culture and Indonesian Maritime Security". Asia and the Pacific Policy Studies 5 (1): 77-89. Disponible en:

https://onlinelibrary.wiley.com/doi/epdf/10.1002/app5.203

Aufiya, Agoes. 2017. "Indonesia's Global Maritime Fulcrum: Contribution in the Indo-Pacific Region”. Andalas Journal of International Studies 6 (2): 143-159. Disponible en:

http://ajis.fisip.unand.ac.id/index.php/ajis/article/download/74/68

Auliya, Putri y Yohanes Sulaiman. 2019. "Indonesia, ASEAN Centrality and Global Maritime Fulcrum". Global \& Strategis 13 (1): 79-90. Disponible en:

https://e-journal.unair.ac.id/JGS/article/view/12205/7161

Booth, Ken. 2014. Navies and Foreign Policy. Oxon: Routledge.

Connelly, Aaron L. 2014. "Indonesian foreign policy under President Jokowi". Sydney. Lowy Institute for International Policy. 1-22. Disponible en: 
https://www.files.ethz.ch/isn/184801/indonesian-foreign-policy-underpresident-jokowi_0.pdf

Damuri, Yose Rizal. 2014. "A Maritime Silk Road and Indonesia's Perspective of Maritime State". Centre for Strategic and International Studies (CSIS) Working Paper Series. Disponible en:

https://www.csis.or.id/uploads/attachments/post/2019/01/10/a_maritim e_silk_road_and_indonesia_s_perspective_of_maritime_state.pdf Elisabeth, Adriana. 2018. "Indonesia's Maritime Vision in the Indian Ocean: Negotiating National Interest and Regional Commitment". En Maritime Governance And South Asia: Trade, Security And Sustainable Development In The Indian Ocean, editado por Schöttli Jivanta, págs. 79-98. Singapur: World Scientific Publishing.

Ford, Michele y Lenore Lyons. 2009. "Fluid Boundaries: Modernity, Nation and Identity in the Riau Islands". En (eds.) Indonesia Beyond the Water's Edge: Managing an Archipelagic State, editado por R. B. Cribb y Michele Ford, págs. 221-238. Singapur: Institute of Southeast Asian Studies Publishing.

Iis Gindarsah. 2015. "Indonesia and the South China Sea: A Two-fold Strategy”. Rajaratnam School of International Studies Commentary 158: 1-3. Disponible en:

https://www.rsis.edu.sg/wp-content/uploads/2015/07/CO15158.pdf

Iis Gindarsah y Adhi Priamarizki. 2015. "Politics, Security, and Defense in Indonesia: The Pursuit of Strategic Autonomy". En Indonesia's Ascent: Power, Leadership, and the Regional Order, editado por Christopher B. Roberts Ahmad D. Habir y Leonard C. Sebastian, págs. 132-154. Nueva York: Palgrave Macmillan.

. 2015. "Indonesia's Maritime Doctrine and Security Concerns". Singapur: Rajaratnam School of International Studies, Nanyang Technological University. Disponible en:

https://www.rsis.edu.sg/wpcontent/uploads/2015/04/PR150409_Indonesias-MaritimeDoctrine.pdf)

Laksmana, Evan. 2014. "Rebalancing Indonesia's naval force”. En Naval Modernisation in South-East Asia: Nature, Causes and Consequences, editado por Geoffrey Till, y Jane Chan, págs. 175-203. Londrés: Routledge. 
2017. "Indonesia Sea Policy: Accelerating Jokowi's Global Maritime Fulcrum?" Washington: The Center for Strategic and International Studies.

Laksmana, Evan A. y Ristian A. Supriyanto. 2018. "Abandoned at Sea: The Tribunal Ruling and Indonesia's Missing Archipelagic Foreign Policy”. Asian Politics \& Policy 10 (2): 300-321.

Mahan, Alferd Thayer. 1890. The Influence of Sea Power Upon History, 1660-1783, Bostón: Little Brown and Company.

Rajaratnam School of International Studies (RSIS). 2014 "Indonesia's Naval Development and Maritime Cooperation". Singapur, National University of Nanyang. Disponible en: https://www.rsis.edu.sg/wpcontent/uploads/2014/07/PR120705_Indonesia_Naval_Development_ Maritime_Cooperation.pdf)

2015. "Indonesia's Global Maritime Fulcrum Challenges and Trajectories". Singapur: Universidad Tecnologica de Nanyang. Disponible en:

https://www.rsis.edu.sg/wp-

content/uploads/2016/07/ER160714_Global-Maritime-Fulcrum.pdf)

Roberts, Christopher B., y Erlina Widyaningsih. 2015. "Indonesia Leadership in ASEAN: Mediation, Agency and Extra-Regional Diplomacy". En Indonesia's Ascent: Power, Leadership, and the Regional Order, editado por Christopher B. Roberts, Ahmad D.Habir, Leonard C. Sebastian, págs. 264-286. Nuevas York: Palgrave Macmillan.

Sa'adah, Kurniawati, Probo Darono Yakti y Siti R. Susanto. 2019. "Evaluating 4 years of Jokowi Sea Toll Policy: The concept of IndonesiaCentric connectivity for economic equality". Masyarakat, Kebudayaan dan Politik 32 (3): 290-302.

Santoso, Dewi y Fadhillah Nafisah. 2017. "Indonesia’s Global Maritime Axis Doctrine: Security Concerns and Recommendations". Jurnal Hubungan Internasional 10 (2): 86-98. Disponible en: https:/ejournal.unair.ac.id/JHI/article/download/7306/4421

Schreer, Benjamin. 2013. "Moving Beyond Ambitions? Indonesia's Military Modernisation". Barton: Australian Strategic Policy Institute. Disponible en: 
https://www.files.ethz.ch/isn/173326/Moving\%20beyond\%20ambitions _\%20Indonesia $\% 27 \mathrm{~s} \% 20$ military $\% 20$ modernisation..pdf)

Schreer, Benjamin. 2015. "Garuda Rising? Indonesia's Arduous Process of Military Change". En Security, Strategy and Military Change in the $21^{\text {st }}$

Century: Cross-Regional Perspectives, editado por Jo Inge Bekkevold, Ian Bowers y Michael Raska, págs. 55-69. Oxon: Routledge.

Scott, David. 2019. "Indonesia Grapples with the Indo-Pacific: Outreach,

Strategic Discourse, and Diplomacy". Journal of Current Southeast Asian Affairs 38 (2): 194-217.

Sebastian, Leonard C., Ristian Atriandi Supriyanto, I. Made Andi Arsana. 2015. "Beyond the Archipelagic Outlook: The Law of the Sea, Maritime Security and the Great Powers". En Indonesia's Ascent: Power, Leadership, and the Regional Order, editado por Christopher B. Roberts, Ahmad D.Habir, Leonard C. Sebastian, págs. 308-334. Nuevas York: Palgrave Macmillan.

Shekhar, Vibhanshu. 2018a. "Is indonesia's 'Indo-Pacific Cooperation' Strategy a Weak Play?". PACNET 47: 1-3. Disponible en: https://www.pacforum.org/sites/default/s3fs-

public/publication/180717_PacNet_47.pdf)

Shekhar, Vibhanshu. 2018b. Indonesia's Foreign Policy and Grand Strategy in the 21st Century: Rise of an Indo-Pacific Power. Oxon: Routledge.

Siwage Dharma Negara y Sanchita Basu Das. 2017. "Challenges for Indonesia to achieve its Maritime Connectivity Plan and Leverage on Regional Initiatives". Singapur: ISEAS - YUSOF ISHAK INSTITUTE. Disponible en:

https://www.iseas.edu.sg/images/pdf/ISEAS_Perspective_2017_3.pdf) Solís Oyarzún, Eri. 1998. "Poderío Marítimo". Revista Marina de Chile 843: $1-16$.

_. 1999. "Algunas Consideraciones sobre la Estrategia Marítima”. Revista Marina de Chile 848: 1-20. $1-7$.

Sriyanto, Nanto. 2018. "Global Maritime Fulcrum, Indonesia-China Growing Relations, and Indonesia's Middelpowermaship in the East Asia Region". Jurnal Kajian Wilayah 9 (1): 1-18. 
Till, Geoffrey. 2013. Seapower: A Guide for the Twenty-First Century. Oxon: Routledge.

Wright, Thomas. 2015. "Middle Powers and the Multilateral Pivot". En MIKTA, Middle Powers, and New Dynamics of Global Governance. The G20 `s Evolving Agenda., editado por Mo Jongryn, págs. 13-31. Nueva York: Palgrave Macmillan. 